\title{
Threshold Manipulation in Parity-Time Symmetric Microresonator Chain
}

\author{
$\underline{\text { S. Phang, }}{ }^{1}$ A. Vukovic, ${ }^{1}$ S. C. Creagh, ${ }^{2}$ T. M Benson, ${ }^{1}$ P. Sewell, ${ }^{1}$ G. Gradoni ${ }^{2}$ \\ ${ }^{1}$ University of Nottingham, George Green Institute for Electromagnetics Research, University Park, \\ Nottingham, NG7 2RD, UK \\ ${ }^{2}$ University of Nottingham, School of Mathematical Sciences, University Park, Nottingham, NG7 2RD, UK \\ sendy.phang@nottingham.ac.uk
}

\begin{abstract}
This paper analyses the eigenfrequencies of an infinitely long chain of microresonators with PT symmetry. The results show that the depth of modulation of the real part of the refractive index can be used to control the threshold point of the PT structure.
\end{abstract}

\section{INTRODUCTION}

The photonic analogue of a quantum system with parity-time (PT) symmetric Hamiltonian could be constructed by having a photonic structure where the spatial profile of the real part of the refractive index has even symmetry while the imaginary part has odd symmetry [1-3]. The odd symmetry of the imaginary refractive index results in structures that have both gain and loss. Interestingly, despite the presence of gain and loss, PT-symmetric structures feature a zero-net power gain or loss if operated before a threshold point, whilst power grows exponentially beyond this threshold point [4]. In lossless structures the eigenfrequencies below the threshold point are purely real, whilst above the threshold point they become complex conjugates indicating the presence of both gain and loss modes. PT symmetric structures based on Bragg gratings, coupled waveguides and cavities have been investigated both theoretically and experimentally, and have demonstrated unidirectional loss-induced invisibility, simultaneous lasing and coherent-absorption and, recently, a loss-induced lasing mode [5-8]. Different applications have been proposed exploiting the behavior at the threshold point, such as optical switching, an optical logical-gate, lasing and memory devices.

In this paper, the band diagrams of eigenfrequencies of an infinite microresonator chain that satisfies PT symmetry are analysed with and without modulation of the real part of resonator refractive index.

\section{PT-CHAIN OF RESONANT CAVITIES}

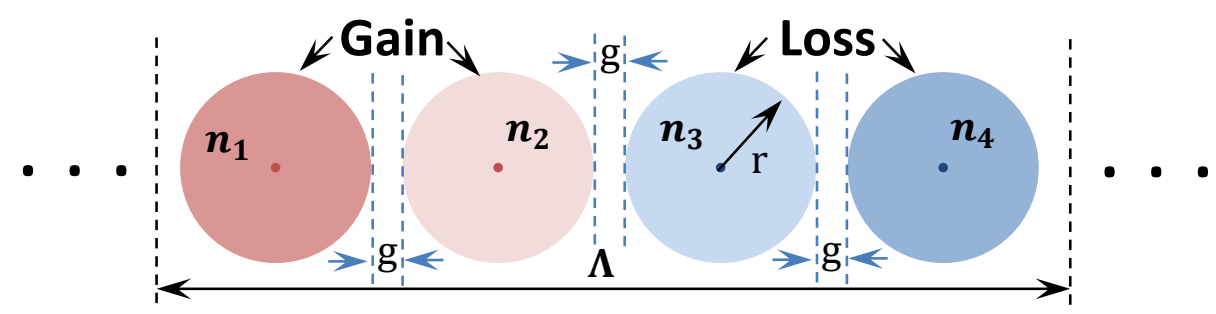

Fig. 1. Schematic illustration of an infinitely long PT microresonator chain

In order to satisfy the requirement that the real part of refractive index has even symmetry, a unit cell is made of four microresonators as shown in Fig.1. A unit cell of length $\Lambda$ is placed in an air background; all resonators have the same radius, $r$ and are separated by the same distance $g$. Refractive indices of individual resonators are,

$$
\left\{\begin{array}{l}
n_{1}=n^{\prime}+\Delta n^{\prime}+j n^{\prime \prime} \\
n_{2}=n^{\prime}-\Delta n^{\prime}+j n^{\prime \prime} \\
n_{3}=n^{\prime}-\Delta n^{\prime}-j n^{\prime \prime} \\
n_{4}=n^{\prime}+\Delta n^{\prime}-j n^{\prime \prime}
\end{array}\right.
$$


where, $n^{\prime}$ denotes the real part of refractive index, $\Delta n^{\prime}$ is the real index modulation and $n^{\prime \prime}$ denotes the imaginary part of the refractive index so that $n^{\prime \prime}>0$ represents gain and $n "<0$ represents loss.

In our analysis, we use an exact representation of the problem based on boundary integral equations which is outlined in [9]. The coupled system can be described in a matrix form as,

$$
\left(\begin{array}{cccc}
\widetilde{D}_{1} & \tilde{C} & 0 & e^{j \beta \Lambda} \tilde{C} \\
\tilde{C} & \widetilde{D}_{2} & \tilde{C} & 0 \\
0 & \tilde{C} & \widetilde{D}_{3} & \tilde{C} \\
e^{-j \beta \Lambda} \tilde{C} & 0 & \tilde{C} & \widetilde{D}_{4}
\end{array}\right)\left(\begin{array}{l}
\tilde{\alpha}_{1} \\
\tilde{\alpha}_{2} \\
\tilde{\alpha}_{3} \\
\tilde{\alpha}_{4}
\end{array}\right)=0
$$

where, elements $\widetilde{D}$ describe the solution of an isolated resonator and elements $\tilde{C}$ describe the coupling between resonators and where $\beta \Lambda$ is the Bloch phase. Solutions of (2) are obtained for four complex eigenfrequencies of the infinite chain. Due to the limited space readers are referred to [9] for details of this method.

\section{RESULTS AND DISCUSSIONS}

An infinite chain with following parameters is considered: $R=0.54 \mu \mathrm{m}, n^{\prime}=3.5$ and $g=0.24 \mu \mathrm{m}$ and operating at the high Q-factor mode $(10,1)$ with resonant frequency $f_{0}=336.85 \mathrm{THz}$ and with the electric field polarized along the resonator axis. Fig. 2, shows the eigenfrequencies of the structure as a function of Bloch phase $(\beta \Lambda)$ for different values of the normalized gain/loss parameter $\gamma_{0}=\omega_{0} n^{\prime \prime}$ A balanced PT system is considered where $\gamma_{0}=-\gamma_{G}=\gamma_{L}$ where subscripts $G$ and $L$ denote gain and loss respectively. Fig. 2(a,b) show the real and imaginary part of eigenfrequencies of the system when the depth of modulation is $\Delta n^{\prime}=0$. It can be seen that the PT system has a complete band diagram, noted by the degenerate modes that occur at $\beta \Lambda=$ 0 and 1 . Figure 2(b) shows that the imaginary part of eigenfrequency split from $\gamma_{0}=0$, denoted by the red line on the 2D surface, indicating that in the case of zero modulation $\left(\Delta n^{\prime}=0\right)$, the PT system is threshold-less.

Figure 2(c,d) shows the real and imaginary parts of the eigenfrequencies when the depth of modulation of the real part of refractive index is $\Delta n^{\prime}=0.0005$. Figure 2(c) shows three notable band-gaps on the eigenfrequency spectrum and marked by red boxes in the figure. Furthermore, as the gain/loss parameter $\gamma_{0}$ increases, the two band-gaps of the real part of the eigenfrequencies reduce and start merging at $\beta \Lambda=\pi$. Meanwhile the imaginary part of the eigenfrequencies, depicted in Fig 2(d), has a distinct threshold line which appears as the band-gaps merge from $\beta \Lambda=\pi$. This indicates that the presence of modulation depth has a significant impact on the appearance of the threshold point. In the presentation we will also include our preliminary results in the case of material dispersion obeying the causality condition.

\section{CONCLUSION}

The results presented show that modulation of the real part of refractive index has significant impact on the appearance of the threshold point of a infinite PT resonator chain. In the absence of the modulation $\Delta n^{\prime}=0$, the PT system is threshold-less. By introducing modulation of the real part of material refractive index $\Delta n^{\prime}>0$, band-gaps are created and, as the gain/loss in the system is decreased the band gaps are reduced; they coalesce at $\beta \Lambda=\pi$ at which the threshold point of the PT system occurs and the imaginary parts of eigenfrequencies start to split. The appearance of the threshold point can thus be controlled in order to better exploit the unique properties of PT systems. 


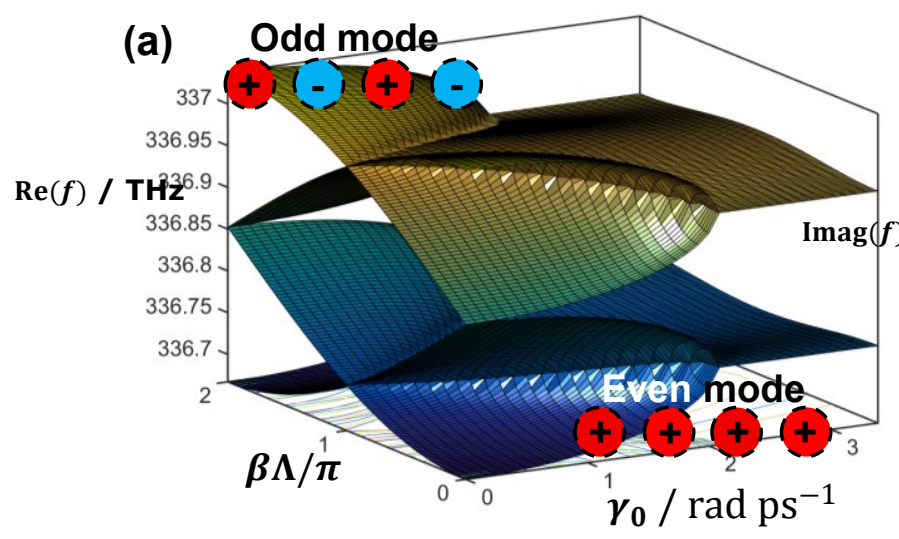

(c)

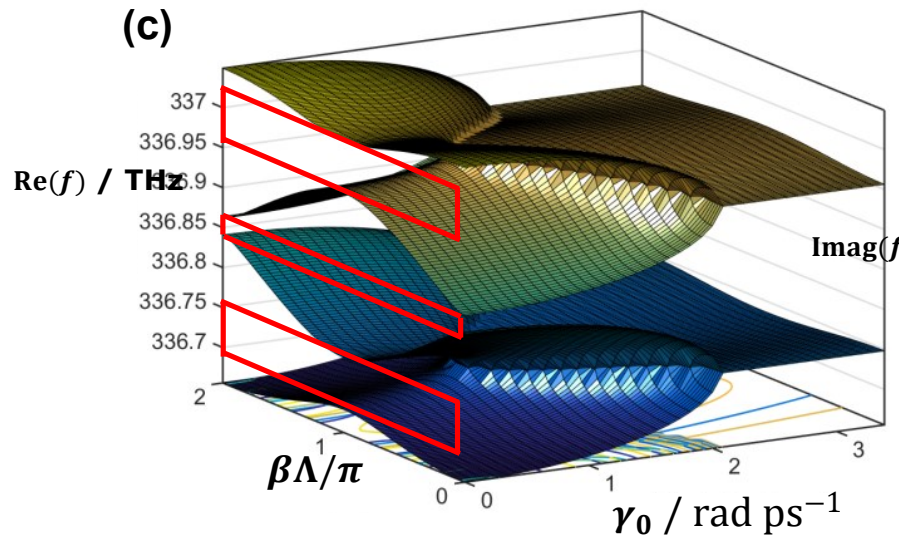

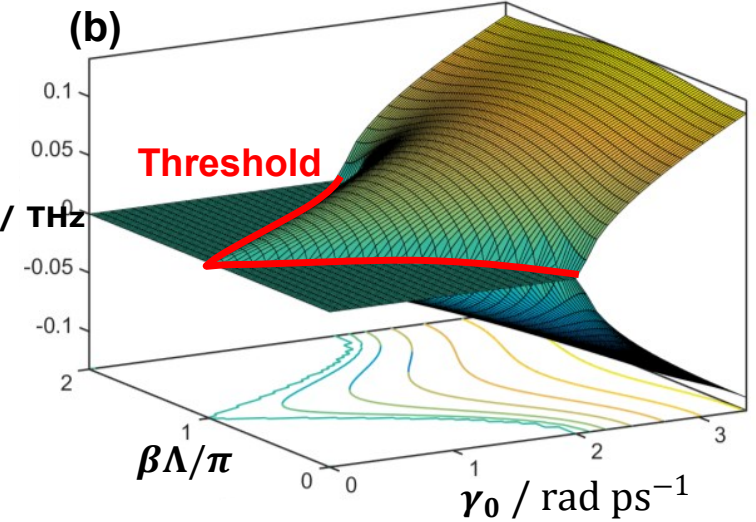

(d)

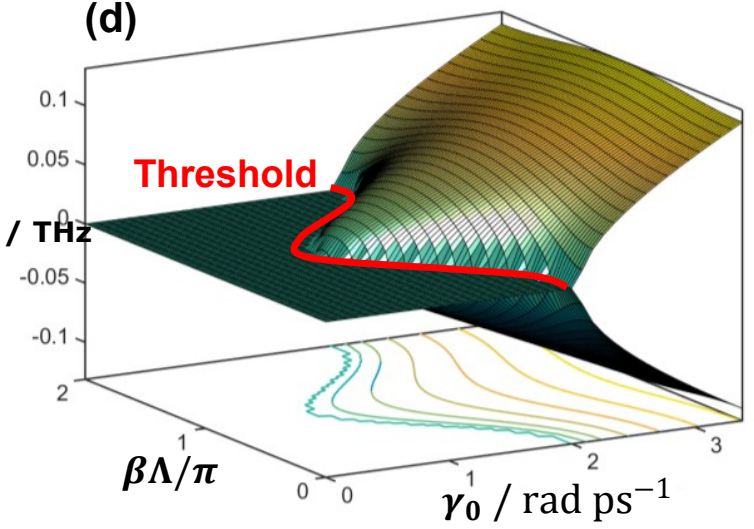

Figure 2 Complex eigenfrequencies of the PT-chain cavities for different values of the gain/loss parameter $\left(\gamma_{0}\right)$ as a function of Bloch phase $(\beta \Lambda)$ for $(\mathrm{a}, \mathrm{b})$ zero modulation of real part of refractive index $\left(\Delta n^{\prime}=0\right)$ and $(\mathrm{c}, \mathrm{d})$ when the index modulation is $\Delta n^{\prime}=0.0005$.

\section{REFERENCES}

1. L. Chang, X. Jiang, S. Hua, C. Yang, J. Wen, L. Jiang, G. Li, G. Wang, and M. Xiao, "Parity-time symmetry and variable optical isolation in active-passive-coupled microresonators," Nat. Photonics 8, 524-529 (2014).

2. Y. D. Chong, L. Ge, and A. D. Stone, "PT-Symmetry Breaking and Laser-Absorber Modes in Optical Scattering Systems," Phys. Rev. Lett. 106, 093902 (2011).

3. B. Peng, Ş. K. Özdemir, F. Lei, F. Monifi, M. Gianfreda, G. L. Long, S. Fan, F. Nori, C. M. Bender, and L. Yang, "Parity-time-symmetric whispering-gallery microcavities," Nat. Phys. 10, 394-398 (2014).

4. S. Phang, A. Vukovic, H. Susanto, T. M. Benson, and P. Sewell, "Ultrafast optical switching using parity-time symmetric Bragg gratings," J. Opt. Soc. Am. B 30, 2984-2991 (2013).

5. S. Phang, A. Vukovic, H. Susanto, T. M. Benson, and P. Sewell, "Impact of dispersive and saturable gain/loss on bistability of nonlinear parity-time Bragg gratings.," Opt. Lett. 39, 2603-6 (2014).

6. J. Čtyroký, "Dispersion properties of coupled waveguides with loss and gain: a full-vectorial analysis," Opt. Quantum Electron. 46, 465-475 (2014).

7. A. Lupu, H. Benisty, and A. Degiron, "Switching using PT symmetry in plasmonic systems: positive role of the losses," Opt. Express 21, 192-195 (2013).

8. B. Peng, H. Yilmaz, M. Liertzer, F. Monifi, C. M. Bender, F. Nori, L. Yang, S. K. Özdemir, S. Rotter, H. Yilmaz, M. Liertzer, F. Monifi, C. M. Bender, F. Nori, L. Yang, . K. Ozdemir, S. Rotter, H. Yilmaz, M. Liertzer, F. Monifi, C. M. Bender, F. Nori, and L. Yang, "Loss-induced suppression and revival of lasing.," Science 346, 328-32 (2014).

9. S. Phang, A. Vukovic, S. C. Creagh, T. M. Benson, P. D. Sewell, and G. Gradoni, "Parity-Time Symmetric Coupled Microresonators with a Dispersive Gain/Loss," accepted for publication Optics Express, arXiv Prepr. arXiv 1501.07455 (2015). 